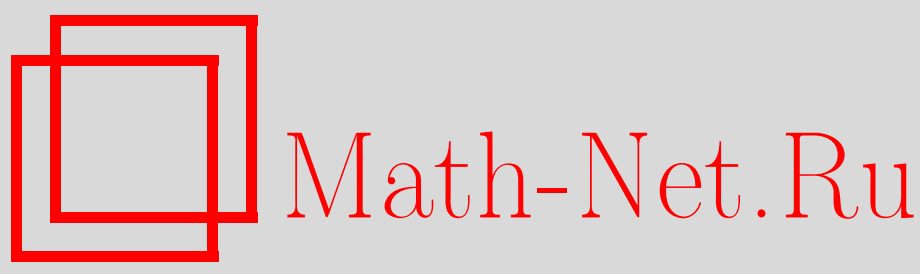

В. Ф. Вильданова, Существование решения задачи Коши для уравнения агрегации в гиперболическом пространстве, Изв. вузов. Матем., 2020, номер 7, 33-44

DOI: https://doi.org/10.26907/0021-3446-2020-7-33-44

Использование Общероссийского математического портала Math-Net.Ru подразумевает, что вы прочитали и согласны с пользовательским соглашением

http://www . mathnet.ru/rus/agreement

Параметры загрузки:

IP: 54.81 .137 .203

26 апреля 2023 г., 18:20:04

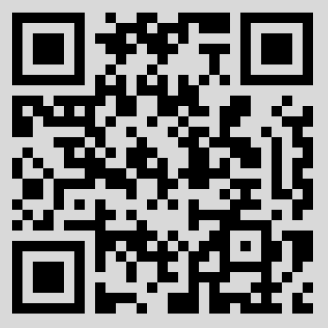


Известия вузов. Математика

2020, № 7 , c. 33-44 https://kpfu.ru/science/nauchnye-izdaniya/ivrm

e-mail: izvuz.matem@kpfu.ru

\title{
В.Ф. ВИЛЬДАНОВА
}

\section{СУЩЕСТВОВАНИЕ РЕШЕНИЯ ЗАДАЧИ КОШИ ДЛЯ УРАВНЕНИЯ АГРЕГАЦИИ В ГИПЕРБОЛИЧЕСКОМ ПРОСТРАНСТВЕ}

\begin{abstract}
Аннотация. В гиперболическом пространстве рассматривается задача Коши для уравнения агрегации. Неотрицательная начальная функция ограничена и суммируема. Доказано существование слабого решения на малом интервале времени. В случае, когда ядро интегрального оператора гладкое и быстро убывает на бесконечности, доказано существование ограниченного решения на произвольном интервале времени.
\end{abstract}

Ключевые слова: уравнение агрегации, существование решения, гиперболическое пространство.

УДК: 517.954

DOI: $10.26907 / 0021-3446-2020-7-33-44$

\section{1. ВВЕДЕНИЕ}

Пусть $\mathbb{H}^{n}$ - гиперболическое пространство размерности $n \geq 2$. В работе [1] исследуется корректность задачи Коши

$$
\begin{gathered}
a(x) u_{t}=\Delta A(u),(x, t) \in \mathbb{H}^{n} \times[0, T), \\
u(x, 0)=u_{0}(x), u_{0} \in C\left(\mathbb{H}^{n}\right) \cap L_{\infty}\left(\mathbb{H}^{n}\right),
\end{gathered}
$$

где $\Delta$ - оператор Лапласа-Бельтрами. Непрерывная функция $a$ положительна, функция $A, A(0)=0$, имеет положительную производную в $\mathbb{R} \backslash\{0\}$. Рассматривается модель Пуанкаре гиперболического пространства $\mathbb{H}^{n}$ в виде шара $B_{1} \subset \mathbb{R}^{n}$ с римановой метрикой $g_{i j}=4 \delta_{i j}\left(1-|x|^{2}\right)^{-2}$. Доказывается существование ограниченного решения задачи Коши. При дополнительном условии $a(x) \geq C(1-|x|)^{-\alpha}, \alpha>0$, доказывается единственность ограниченного решения задачи Коши. Если же $a(x) \leq C(1-|x|)^{-\alpha}, \alpha<0$, то решение задачи Коши имеет след на бесконечности, и для единственности нужно задавать значение этого следа.

Мотивацией для настоящего исследования явились работы [1], [2]. В [2] доказывается существование и единственность ограниченного решения задачи Коши для уравнения агрегации

$$
u_{t}=\triangle A(u)-\operatorname{div}(u \nabla K * u)
$$

Поступила в редакцию 17.06.2019, после доработки 20.12.2019. Принята к публикации 25.03.2020.

Благодарности. Работа выполнена при финансовой поддержке Российского фонда фундаментальных исследований № 18-01-00428а. 
с гладким симметричным ядром $K=K(|x|)$. Была доказана также корректность первой смешанной задачи в цилиндре $D^{T}=\Omega \times(0, T)$ в случае выпуклой области $\Omega \subset \mathbb{R}^{n}$. Однако доказательства существования решения содержали пробел, который был устранен в работе [3].

Работу [1] можно рассматривать как распространение результатов работы [4] о корректности задачи Коши для уравнения

$$
a(x) u_{t}=\triangle u, \quad x \in \mathbb{R}^{n}, t>0,
$$

на случай гиперболического пространства. В этой работе доказано, что если функция $a(x)$ достаточно быстро убывает на бесконечности, то ограниченное решение задачи Коши для уравнения (3) имеет определенный след на бесконечности в виде функции времени $\gamma(t)$, и для единственности решения нужно задавать значение этого следа.

Отметим, что уравнения (1), (2) существенно различны. Если для уравнений (1), (3) справедлив принцип максимума в обычной для параболического уравнения форме, то для уравнения (2) принцип максимума неизвестен (и, по-видимому, не имеет места). Поэтому методы работы с уравнениями $(1),(2)$ сильно различаются.

В настоящей работе в слое $Q^{T}=\mathbb{H}^{n} \times[0, T)$ рассматривается задача Коши для уравнения агрегации

$$
u_{t}=\operatorname{div}_{g}(a(u)-u \mathcal{G}(u)),
$$

где $a(u)=\left|\nabla_{g} A(u)\right|_{g}^{p-2} \nabla_{g} A(u)$, с ограниченной начальной функцией $u_{0} \in L_{1}\left(\mathbb{H}^{n}\right)$ :

$$
u(x, 0)=u_{0}, \quad u_{0}(x) \geq 0, \quad x \in \mathbb{H}^{n} .
$$

Здесь и далее у операторов $\operatorname{div}_{g}, \nabla_{g}$ нижний индекс $g$ на многообразии $\mathbb{H}^{n}$ будет отличать их от соответствующих операторов в $\mathbb{R}^{n}$. Пусть $B_{r}=\left\{x \in \mathbb{R}^{n}|| x \mid<r\right\}$ - шар радиуса $r \in(0,1]$. Условия на интегральный оператор

$$
\mathcal{G}(v)=\int_{\mathbb{H}^{n}} X(y) v(y) d \nu
$$

приведены в секции 2 ; здесь $d \nu$ - элемент объема в $\mathbb{H}^{n}, X(y)$ при каждом $y \in \mathbb{H}^{n}$ является векторным полем в $\mathbb{H}^{n}$.

Обзор работ, посвященных вопросам существования и единственности решения смешанной задачи для уравнения агрегации в случае областей в $\mathbb{R}^{n}$, можно найти в [3]. Отметим, что в последнее десятилетие проводится очень много исследований, касающихся уравнения агрегации. Соответствующие ссылки можно найти в списках литературы работ [2], [5].

\section{2. ОБОЗНАЧЕНИЯ, ОПРЕДЕЛЕНИЯ, РЕЗУЛЬТАТ}

Будем рассматривать гиперболическое пространство $\mathbb{H}^{n}$ в виде модели Пуанкаре в шаре $B_{1}$ с римановой метрикой

$$
g_{i j}(x)=\frac{4}{\left(1-|x|^{2}\right)^{2}} \delta_{i j}, \quad x \in B_{1}, \quad i, j=1, \ldots, n
$$

кроме того, пусть $g^{i j}-$ элементы обратной матрицы к $\left(g_{i j}\right)$,

$$
\partial B_{1} \equiv \partial_{\infty} \mathbb{H}^{n}=\{\infty\} .
$$


Геодезическое расстояние между произвольным $x \in \mathbb{H}^{n}$ и точкой 0 определяется формулой

$$
\rho(x)=\int_{0}^{|x|} \frac{2}{1-s^{2}} d s=\ln \frac{1+|x|}{1-|x|}, \quad x \in \mathbb{H}^{n} \equiv B_{1} ;
$$

поэтому

$$
|x|=\tanh \left(\frac{\rho(x)}{2}\right)
$$

Для любого $\varrho>0$ определим

$$
\mathfrak{B}_{\varrho}=\left\{x \in \mathbb{H}^{n} \mid \rho(x)<\varrho\right\}, \quad \mathfrak{S}_{\varrho}=\left\{x \in \mathbb{H}^{n} \mid \rho(x)=\varrho\right\} .
$$

Следовательно, для любого $r \in(0,1)$

$$
B_{r}=\mathfrak{B}_{\ln \frac{1+r}{1-r}} .
$$

Всюду далее числа $r, \varrho$ связаны равенством $\varrho=\ln \frac{1+r}{1-r}$. Ниже будем использовать обозначения $D_{r}^{T}=B_{r} \times[0, T), Q_{r}^{T}=\mathfrak{B}_{\varrho} \times[0, T)$.

$\mathrm{B} \mathbb{H}^{n}$ элемент объема имеет вид

$$
d \nu=\sqrt{g} d x_{1} d x_{2} \cdots d x_{n}=\frac{2^{n}}{\left(1-|x|^{2}\right)^{n}} d x,
$$

где $d x$ - мера Лебега в $\mathbb{R}^{n}$.

Для каждого $x \in \mathbb{H}^{n}$ через $T_{x} \mathbb{H}^{n}$ обозначим касательное пространство в этой точке. Очевидно, $\left(\frac{\partial}{\partial x_{1}}, \frac{\partial}{\partial x_{2}}, \ldots, \frac{\partial}{\partial x_{n}}\right)$ - базис в $T_{x} \mathbb{H}^{n}$. Скалярное произведение векторов будем обозначать в угловых скобках

$$
\langle\beta, \xi\rangle_{g} \equiv\langle\beta, \xi\rangle_{g, x}=\sum_{i, j=1}^{n} g_{i j}(x) \beta^{i} \xi^{j}
$$

для любых $\beta, \xi \in T_{x} \mathbb{H}^{n}$, где $\beta=\sum_{i=1}^{n} \beta^{i} \frac{\partial}{\partial x_{i}}, \xi=\sum_{i=1}^{n} \xi^{i} \frac{\partial}{\partial x_{i}}$ для некоторых $\left(\beta^{1}, \ldots, \beta^{n}\right) \in R^{n}$ и $\left(\xi^{1}, \ldots, \xi^{n}\right) \in R^{n}$. Градиент $\nabla_{g} u=\left(\left(\nabla_{g} u\right)^{1}, \ldots,\left(\nabla_{g} u\right)^{n}\right)$ определяется формулой

$$
\left(\nabla_{g} u\right)^{i}=\sum_{j=1}^{n} g^{i j} \frac{\partial u}{\partial x_{j}}, \quad i=1, \ldots, n,
$$

кроме того,

$$
|\beta|_{g}=\sqrt{\langle\beta, \beta\rangle_{g}}, \quad \beta \in T_{x} \mathbb{H}^{n}, \quad x \in \mathbb{H}^{n}
$$

Легко видеть, что

$$
\nabla_{g} A(u)=\frac{\left(1-|x|^{2}\right)^{2}}{4} \nabla A(u), \quad\left|\nabla_{g} A(u)\right|_{g}=\frac{\left(1-|x|^{2}\right)}{2}|\nabla A(u)|,
$$

где $\nabla$ без индекса - обычный градиент в декартовой системе в $\mathbb{R}^{n}$.

Через $\chi^{k}(\mathcal{M})$ обозначим множество векторных полей класса $C^{k}, k \geq 0$, на многообразии $\mathcal{M}$. 
Дифференциал $d f$ функции $f$ имеет локальные координаты $\frac{\partial f}{\partial x^{i}}$, при этом $|d f|_{g}=\left|\nabla_{g} f\right|_{g}$. Производная Ли функции $f \in C^{1}\left(\mathbb{H}^{n}\right)$ вдоль векторного поля $X \in \chi^{0}\left(\mathbb{H}^{n}\right)$ в локальной системе координат определяется формулой

$$
\langle d f, X\rangle=\left\langle\nabla_{g} f, X\right\rangle_{g}=X^{i} \frac{\partial f}{\partial x^{i}} .
$$

Дивергенция векторного поля $X$ в локальной системе координат имеет вид

$$
\operatorname{div}_{g} X=\frac{1}{\sqrt{g}} \frac{\partial}{\partial x^{i}}\left(X^{i} \sqrt{g}\right)
$$

Пространство $\stackrel{\circ}{W}_{p}^{1,0}\left(Q_{r}^{T}\right)$ определяется как пополнение пространства $C_{0}^{1}\left(Q_{r}^{T}\right)$ по норме

$$
\|u\|_{W_{p}^{1,0}\left(Q_{r}^{T}\right)}^{p}=\|u\|_{p, Q_{r}^{T}}^{p}+\left\|\nabla_{g} u\right\|_{p, Q_{r}^{T}}^{p}=\int_{0}^{T} \int_{\mathfrak{B}_{\varrho}}\left(|u|^{p}+\left|\nabla_{g} u\right|_{g}^{p}\right) d \nu d t .
$$

Поскольку

$$
1-r^{2} \leq 1-|x|^{2} \leq 1, \quad x \in B_{r}, r \in(0,1),
$$

то $\stackrel{\circ}{W}_{p}^{1,0}\left(Q_{r}^{T}\right)$ совпадает с обычным соболевским пространством $\stackrel{\circ}{W}_{p}^{1,0}\left(D_{r}^{T}\right)$ с нормой

$$
\|u\|_{W_{p}^{1,0}\left(D_{r}^{T}\right)}=\left(\int_{0}^{T} \int_{B_{r}}\left(|u|^{p}+|\nabla u|^{p}\right) d x d t\right)^{1 / p}
$$

и соответствующие нормы эквивалентны в силу (6). Пространство $W_{p}^{1,0}\left(Q_{r}^{T}\right)$ определяется как пополнение пространства $C^{1}\left(\overline{Q_{r}^{T}}\right)$.

Будем предполагать, что $A(u) \in C^{1}[0, \infty), A(0)=0$, и

$$
A^{\prime}(u)>0, u>0 \text {. }
$$

Условия на ядро оператора $\mathcal{G}(v)$ имеют вид $X(y) \in \chi^{1}(P), P=\mathbb{H}^{n} \backslash\{y\}, y \in B_{1}$,

$$
\left|d_{x} X_{x}(y)\right|_{g}+\left|X_{x}(y)\right| \leq C(|x|)\left(1+|x-y|^{-\lambda}\right), \quad \lambda \in(0, n), \quad x, y \in B_{1},
$$

где $C(r)$ - неубывающая функция. В следующем условии дивергенция вычисляется в естественной карте $B_{1}$ :

$$
\operatorname{div}_{x}\left(\sqrt{g(x)} X_{x}(y)\right) \geq-D_{X}, \quad x, y \in B_{1} .
$$

Условию (9) удовлетворяют, например, гладкие векторные поля $X_{x}(y)$, достаточно быстро убывающие при $|x| \rightarrow 1$.

Из условия $\lambda \in(0, n)$ следует, что найдется число $q \geq 2$ такое, что $\lambda<\frac{n}{\bar{q}}, \frac{1}{q}+\frac{1}{\bar{q}}=1$. Зафиксируем соответствующие числа $q, \bar{q}$.

Будем использовать следующее утверждение об оценках интегралов типа потенциалов ([6], гл. I, §6).

Лемма 1. Пусть $\lambda \in(0, n) u \lambda<n / \bar{q}, 2 \leq q<\infty, f(x) \in L_{q}\left(B_{1}\right)$. Тогда функиия

$$
v(x)=\int_{B_{1}} \frac{f(y) d y}{|x-y|^{\lambda}}
$$

непрерывна в $B_{1}$ и удовлетворяет неравенству $|v(x)| \leq C\|f\|_{q, B_{1}}, x \in B_{1}$. 
Выведем некоторые оценки для интегрального оператора $\mathcal{G}(v)$. Из леммы 1 и условия (8) следует, что для всякой измеримой функции $v(x)$ с носителем $B_{r},|v(x)| \leq \rho$, выполнены неравенства

$$
\mathcal{G}(v) \in \chi^{1}\left(\mathbb{H}^{n}\right), \quad|\mathcal{G}(v)(x)| \leq C_{G}(\rho),\left|\nabla_{g} \mathcal{G}(v)(x)\right|_{g} \leq C_{d}(\rho), \quad x \in B_{r},
$$

с возрастающими функциями $C_{G}, C_{d}$, которые могут зависеть от $r$.

Определение 1. Функция $u: Q^{T} \rightarrow[0, \infty)$ называется слабым решением задачи Коши (4), $(5)$, если $u \in L_{\infty}\left(Q^{T}\right), A(u) \in W_{p}^{1,0}\left(Q_{r}^{T}\right)$ при любом $r \in(0,1)$ и для всех пробных функций $\phi \in C_{0}^{\infty}\left(Q^{T}\right)$ выполнено равенство

$$
\int_{Q^{T}}\left(\left(u_{0}-u\right) \phi_{t}+\left\langle\left|\nabla_{g} A(u)\right|_{g}^{p-2} \nabla_{g} A(u), d \phi\right\rangle\right) d \nu d t=\int_{Q^{T}}\langle u \mathcal{G}(u), d \phi\rangle d \nu d t .
$$

Теорема 1. Пусть $0 \leq u_{0}(x) \leq M_{0}, u_{0} \in L_{1}\left(\mathbb{H}^{n}\right)$ и выполнены условия (7), (8). Тогда существует слабое решение задачи (4), (5) в иилиндре $Q_{r}^{T}$ малой высоты $T$.

Если дополнительно выполнено условие (9), то существует слабое решение задачи в цилиндре $Q^{T}$ любой высоты $T>0$.

\section{3. ДОКАЗАТЕЛЬСТвО ТЕОРЕМЫ 1}

Не ограничивая общности будем считать, что $A(r)$ - нечетная функция. Рассмотрим аппроксимационную задачу. Положим

$$
A_{\varepsilon}(u)=A(u)+\varepsilon u, \quad a_{\varepsilon}(u)=\left|\nabla_{g} A_{\varepsilon}(u)\right|_{g}^{p-2} \nabla_{g} A_{\varepsilon}(u) .
$$

Шар $B_{r}=\left\{x \in \mathbb{R}^{n}|| x \mid<r\right\}, r \in(0,1)$, является естественной картой для геодезического шара $\mathfrak{B}_{\varrho} \subset \mathbb{H}^{n}$ радиуса $\varrho=\frac{1+r}{1-r}$. В шаре $\mathfrak{B}_{\varrho}$ будем рассматривать уравнение

$$
u_{t}=\operatorname{div}_{g}\left(a_{\varepsilon}(u)-u \mathcal{G}(u)\right), \quad x \in \mathfrak{B}_{\varrho}, \quad t \in(0, T),
$$

с начальным и краевым условиями

$$
\begin{gathered}
u(x, 0)=u_{0}, \quad u_{0}(x) \geq 0, \quad x \in \mathfrak{B}_{\varrho}, \\
\left.u\right|_{x \in \partial \mathfrak{B}_{\varrho}}=0 .
\end{gathered}
$$

При вычислении интегрального оператора $\mathcal{G}(u)$ предполагается, что функция $u$ продолжена нулем вне $Q_{r}^{T}$.

Чтобы установить разрешимость задачи (11)-(13), перепишем уравнение (11) в карте $B_{r}$. В силу (6) имеем

$$
\begin{aligned}
\operatorname{div}_{g}\left(a_{\varepsilon}(u)\right)= & \frac{1}{\sqrt{g}} \operatorname{div}\left(\sqrt{g} \frac{\left(1-|x|^{2}\right)^{p}}{2^{p}}\left|\nabla A_{\varepsilon}(u)\right|^{p-2} \nabla A_{\varepsilon}(u)\right), \\
& \operatorname{div}_{g}(u \mathcal{G}(u))=\frac{1}{\sqrt{g}} \operatorname{div}(\sqrt{g} u \mathcal{G}(u)) .
\end{aligned}
$$

Тогда задача (11)-(13) сводится к следующей задаче в шаре $B_{r} \subset \mathbb{R}^{n}$ :

$$
\begin{gathered}
\sqrt{g} u_{t}=\operatorname{div}\left(\sqrt{g} \frac{\left(1-|x|^{2}\right)^{p}}{2^{p}}\left|\nabla A_{\varepsilon}(u)\right|^{p-2} \nabla A_{\varepsilon}(u)-\sqrt{g} u \mathcal{G}(u)\right), x \in B_{r}, t \in(0, T), \\
u(x, 0)=u_{0}, \quad u_{0}(x) \geq 0, \quad x \in B_{r}, \\
\left.u\right|_{x \in \partial B_{r}}=0 .
\end{gathered}
$$

Существование слабого решения задачи (14)-(16) выведем из результата работы [7]. 
После обозначений $\widehat{a}(x, u, \nabla u)=\sqrt{g} \frac{\left(1-|x|^{2}\right)^{p}}{2^{p}}\left|\nabla A_{\varepsilon}(u)\right|^{p-2} \nabla A_{\varepsilon}(u), G(u)=\sqrt{g} \mathcal{G}(u)$, уравнение (14) перепишется в виде

$$
\sqrt{g} u_{t}=\operatorname{div}(\widehat{a}(x, u, \nabla u)-u G(u)) .
$$

Очевидно, существует непрерывная функция $C(m, r), m \geq 0, r \in(0,1)$, такая, что

$$
|\widehat{a}(x, s, y)|^{\bar{p}} \leq C(m, r)|y|^{p}
$$

при всех $s \in[-m, m], y \in \mathbb{R}^{n}, x \in B_{r}$. Отметим, что из (18) легко следует

$$
|\widehat{a}(x, u, \nabla v)| \in L_{\bar{p}}\left(D_{r}^{T}\right)
$$

при всех $u \in L_{\infty}\left(D_{r}^{T}\right), v \in W_{p}^{1,0}\left(D_{r}^{T}\right)$.

Определение 2. Функция $u: D_{r}^{T} \rightarrow[0, \infty)$ называется слабым решением задачи (17), $(15),(16)$ в цилиндре $D_{r}^{T}$, если $u \in L_{\infty}\left(Q^{T}\right), u \in \stackrel{\circ}{p}_{p}^{1,0}\left(D_{r}^{T}\right)$ и для всех пробных функций $\phi \in C_{0}^{\infty}\left(D_{r}^{T}\right)$ выполнено равенство

$$
\int_{D_{r}^{T}}\left(\sqrt{g}\left(u_{0}-u\right) \phi_{t}+(\widehat{a}(x, u, \nabla u)-u G(u)) \cdot \nabla \phi\right) d x d t=0 .
$$

Функция $u$ является решением в $D_{r}^{\infty}=B_{r} \times[0, \infty)$, если она является решением в $D_{r}^{T}$ при любом $T>0$.

Теорема 2. Пусть $0 \leq u_{0}(x) \leq M_{0}$ и выполнены условия (7), (8). Тогда существует слабое решение задачи (17), (15), (16) в цилиндре $D_{r}^{T}$ малой высоты $T$ такое, что $0 \leq u(x, t) \leq \rho$.

Если, дополнительно, выполнено условие (9), то существует слабое решение задачи (17), (15), (16) в изииндре $D_{r}^{\infty}$ такое, что $0 \leq u(x, t) \leq M_{0} \exp (c t)$, с не зависит от $r$.

Лемма 2. Пусть $\rho \geq 3 M_{0}$. Тогда найдется число $T=T\left(C_{d}(\rho), C_{G}(\rho)\right)$ такое, что существует решение задачи (17), (15), (16) в иилиндре $D_{r}^{T}$ такое, что $0 \leq u(x, t) \leq \rho$.

Доказательство этой леммы фактически следует из результата работы [7] (см. также [8]), поэтому опускается. В этой работе рассматривалась задача с краевым условием «непротекания». В случае краевого условия (16) доказательство упрощается.

Лемма 3. Пусть $b(s)$ - функиия, неубывающал по $s$, и измеримые функции $v: Q^{T} \rightarrow \mathbb{R}$, $v_{0}: \mathbb{H} \rightarrow \mathbb{R}$ таковы, что $b(v) \in L_{1}\left(Q_{r}^{T}\right), b\left(v_{0}\right) \in L_{1}\left(B_{r}\right)$. Пусть $w \in\left(W_{p}^{1,0}\left(Q_{r}^{T}\right)\right)^{\prime}+L_{1}\left(Q_{r}^{T}\right) u$

$$
\int_{Q^{T}} \varphi_{t}\left(b(v)-b\left(v_{0}\right)\right) d \nu d t+(w, \varphi)_{Q_{r}^{T}}=0
$$

при всех $\varphi \in C_{0}^{1}\left(Q_{r}^{T}\right)$.

Тогда

$$
-\left(b(v)_{t}, \xi(v) \varphi\right)_{Q^{T}}=\int_{Q^{T}} \varphi_{t} \int_{v_{0}}^{v} \xi(r) d b(r) d \nu d t
$$

при всех ограниченных $\xi(s)$, монотонных и липшицевых таких, что $\xi(v) \in \stackrel{\circ}{W}_{p}^{1,0}\left(Q_{r}^{T}\right), u$ $\varphi \in C_{0}^{1}\left(Q^{T}\right)$. 
Доказательство, проведенное в [9] (см. также [5]) для цилиндра $D^{T}$, без существенных изменений переносится на случай многообразий.

Докажем следующее утверждение. Если решение $u$ задачи (17), (15), (16) существует в цилиндре $D_{r}^{T^{\prime}}$, то существует решение $v$ в цилиндре $D_{r}^{T^{\prime}+\varepsilon\left(T^{\prime}\right)}$, совпадающее с $u$ в цилиндре любой высоты $T<T^{\prime}$.

Лемма 4. Пусть функиия $u(x, t)$ - слабое решение задачи (17), (15), (16) в иилиндре $D_{r}^{T^{\prime}}$. Тогда при всех $t \in\left[0, T^{\prime}\right]$

$$
\int_{B_{r}} \sqrt{g} u(x, t) d x \leq \int_{B_{r}} \sqrt{g} u_{0}(x) d x .
$$

Доказательство. Подставляя в (19) функцию $\phi=\varphi(t) \psi(x)$, где $\varphi \in C_{0}^{1}\left[0, T^{\prime}\right), \psi \in C_{0}^{1}\left(B_{r}\right)$, получаем

$-\int_{0}^{T^{\prime}} \varphi^{\prime}(t) \int_{B_{r}} \sqrt{g} u(x, t) \psi d x d t=\int_{B_{r}} \sqrt{g} u_{0}(x) \varphi(0) \psi d x+\int_{0}^{T^{\prime}} \varphi(t) \int_{B_{r}}(u G(u)-\widehat{a}(x, u, \nabla u)) \cdot \nabla \psi d x d t$.

Отсюда следует абсолютная непрерывность по $t$ функции $\int_{B_{r}} \sqrt{g} u(x, t) \psi d x d t$, слабая непрерывность $\sqrt{g} u(x, t) \in C_{w}\left(\left[0, T^{\prime}\right) ; L_{2}\left(B_{r}\right)\right)$ и равенство $u(0)=u_{0}$.

Пусть $H(r)=\min (1, \max (r, 0))$. Пользуясь леммой 3 , подставим в (19) пробную функцию $\phi=H\left(\varepsilon^{-1} u\right) H(m(\tau-t)) \psi$, где $\psi(x)=1$ при $x \in B_{r}, \psi \in C_{0}^{1}\left(Q^{T}\right)$. Поскольку $\widehat{a} \cdot \nabla H\left(\varepsilon^{-1} u\right)=$ $=\varepsilon^{-1} \chi(0<u<\varepsilon) \widehat{a} \cdot \nabla u \geq 0$, то после предельного перехода $m \rightarrow \infty$ будем иметь

$$
\begin{gathered}
\int_{B_{r}} \sqrt{g} \int_{u_{0}}^{u(\tau)} H\left(\frac{s}{\varepsilon}\right) d s d x \leq \frac{1}{\varepsilon} \int_{0}^{\tau} \int_{B_{r}} u G(u) \cdot \nabla u \chi(0<u<\varepsilon) d x d t \leq \\
\leq \int_{0}^{\tau} \int_{B_{r}} \chi(0<u<\varepsilon)|G(u) \cdot \nabla u| d x d t .
\end{gathered}
$$

Так как мера множества $\{(x, t) \mid 0<u(x, t)<\varepsilon\}$ стремится к нулю при $\varepsilon \rightarrow 0$, то интеграл в правой части исчезает после предельного перехода в (20). Поэтому

$$
\int_{B_{r}} \sqrt{g} \int_{u_{0}}^{u(x, \tau)} \chi(s>0) d s d x \leq 0 .
$$

Пользуясь условием (9) и леммой 4 , находим

$-\operatorname{div} G(u)=-\int_{B_{r}} \operatorname{div}(\sqrt{g(x)} X(y)) u(y, t) \sqrt{g(y)} d y \leq \int_{B_{r}} D_{X} \sqrt{g(y)} u(y, t) d y \leq D_{X} \int_{B_{r}} \sqrt{g} u_{0}(y) d y \leq \mu$,

где $\mu$ не зависит от $r$.

Лемма 5. Пусть и-решение задачи (17), (15), (16) в $D_{r}^{T^{\prime}}$. Тогда функция $u(x, t)$ неотрицательна и

$$
\|u(t)\|_{L_{\infty}\left(B_{r}\right)} \leq e^{\mu t}\left\|u_{0}\right\|_{L_{\infty}\left(B_{r}\right)} .
$$


Доказательство. Сделав замену $u=v e^{\mu t}$ в тождестве (19), получим

$$
\int_{D_{r}^{T^{\prime}}}\left(\sqrt{g}\left(u_{0}-v e^{\mu t}\right) \phi_{t}+\nabla \phi \cdot\left(\widehat{a}(u)-v e^{\mu t} G(u)\right)\right) d x d t=0 .
$$

После интегрирования по частям это соотношение примет вид

$$
\left(\sqrt{g} v_{t}, e^{\mu t} \phi\right)_{D_{r}^{T^{\prime}}}+\int_{D_{r}^{T^{\prime}}}\left(\mu v e^{\mu t} \phi+\nabla \phi \cdot\left(\widehat{a}(u)-v e^{\mu t} G(u)\right)\right) d \nu d t=0 .
$$

Подставим сюда $\phi=e^{-\mu t} v^{(k)}, v^{(k)}=\max (0, v-k)$. Учитывая, что

имеем

$$
\nabla \phi \cdot \widehat{a}(u)=e^{-\mu t} \nabla v^{(k)} \cdot \sqrt{g} \frac{\left(1-|x|^{2}\right)^{p}}{2^{p}}\left|\nabla A_{\varepsilon}(u)\right|^{p-2} \nabla A_{\varepsilon}(u) \geq 0
$$

$$
\left(v_{t}, v^{(k)}\right)_{D_{r}^{T^{\prime}}}+\int_{D_{r}^{T^{\prime}}}\left(\mu v^{(k)} v-\nabla v^{(k)} \cdot v G(u)\right) d x d t \leq 0 .
$$

Заметим, что $v^{(k)} v_{t}=v^{(k)}\left(v^{(k)}+k\right)_{t}=v^{(k)} v_{t}^{(k)}, v \nabla v^{(k)}=\left(v^{(k)}+k\right) \nabla v^{(k)}=\nabla\left(\left(v^{(k)}\right)^{2} / 2+k v^{(k)}\right)$. Поэтому в силу $(21)$

$$
\int_{D_{r}^{T^{\prime}}} \nabla v^{(k)} \cdot v G(u) d x d t=-\int_{D_{r}^{T^{\prime}}}\left(\left(v^{(k)}\right)^{2} / 2+k v^{(k)}\right) \operatorname{div} G(u) d x d t \leq \mu \int_{D_{r}^{T^{\prime}}}\left(\left(v^{(k)}\right)^{2} / 2+k v^{(k)}\right) d x d t .
$$

Тогда из (22), выбрав $k=\|v(0)\|_{L_{\infty}\left(B_{r}\right)}, v^{(k)}(0)=0$, получаем

$$
\int_{B_{r}} \sqrt{g}\left(v^{(k)}\left(T^{\prime}\right)\right)^{2} / 2 d x+\mu \int_{D_{r}^{T^{\prime}}}\left(v v^{(k)}-\left(v^{(k)}\right)^{2} / 2-k v^{(k)}\right) d x d t \leq 0 .
$$

Отсюда $v^{(k)} \equiv 0$ или $v \leq k$.

Пусть решение задачи существует в цилиндре $D_{r}^{T^{\prime}}$. Положим

$$
\rho=3 \exp \left(\mu T^{\prime}\right) M_{0}, \quad \widetilde{M}_{0}=\rho / 3 .
$$

По лемме 5 имеем неравенство $\|u\|_{\infty, D_{r}^{T^{\prime}}} \leq \rho / 3$. Пусть $T_{1}=T\left(C_{d}(\rho), C_{G}(\rho)\right)=2 \varepsilon$ определяется как в лемме 2, и $v$ - решение задачи $(17),(15),(16)$ в цилиндре $\left(D_{r}\right)_{T^{\prime \prime}}^{T^{\prime \prime}+T_{1}}, T^{\prime \prime} \in\left(0, T^{\prime}\right)$, с начальной функцией $v\left(x, T^{\prime \prime}\right)=u\left(x, T^{\prime \prime}\right)$. Существование этого решения следует из леммы 2. Запишем для $v$ тождество (19) с пробной функцией $\phi \in C_{0}^{1}\left(D_{r}^{T^{\prime \prime}+T_{1}}\right)$ в виде

$$
\int_{B_{r} \times\left(T^{\prime \prime}, T^{\prime \prime}+T_{1}\right)}\left(-v \phi_{t}+\nabla \phi \cdot(\widehat{a}(v)-v G(v))\right) d x d t=\int_{B_{r}} u\left(x, T^{\prime \prime}\right) \phi\left(x, T^{\prime \prime}\right) d x .
$$

Пользуясь слабой непрерывностью функции $u$, из (19) для $u$ нетрудно вывести равенство

$$
\int_{D_{r}^{T^{\prime \prime}}}\left(-u \phi_{t}+\nabla \phi \cdot(\widehat{a}(u)-u G(u))\right) d x d t=-\int_{B_{r}} u\left(x, T^{\prime \prime}\right) \phi\left(x, T^{\prime \prime}\right) d x+\int_{B_{r}} u_{0}(x) \phi(x, 0) d x .
$$

Доопределим функцию $v$, полагая $v=u$ в $D_{r}^{T^{\prime \prime}}$. Складывая (23), (24) устанавливаем, что $v$ является решением задачи в $D_{r}^{T^{\prime \prime}+T_{1}}$. Выбирая $T^{\prime \prime}$ так, чтобы $T^{\prime \prime}+T_{1}>T^{\prime}+\varepsilon$, устанавливаем справедливость сформулированного перед леммой 4 утверждения. 
Пусть $\widetilde{v}$ - решение задачи в цилиндре $D_{r}^{\widetilde{T}}$, совпадающее с решением $u$ при $t \leq T^{\prime}-\delta$, $\delta>0$. Пусть $T^{*}=\sup \widetilde{T}$ по всем таким $\widetilde{v}$. Покажем, что $T^{*}=\infty$. От противного, если $T^{*}$ - конечное число, то существует $\widetilde{T}>T^{*}-\varepsilon\left(T^{*}\right)$. По доказанному утверждению существует решение задачи $\bar{v}$ в цилиндре $D_{r}^{\widetilde{T}+\varepsilon\left(T^{*}\right)}$, совпадающее с $\widetilde{v}$ при $t \leq \widetilde{T}-\delta$. Неравенство $\widetilde{T}+\varepsilon\left(T^{*}\right)>T^{*}$ противоречит определению супремума. Легко видеть, что тогда существует решение задачи в $D_{r}^{\infty}$. Теорема 2 доказана.

Решение задачи (11)-(13) при $r=1-\varepsilon$, существование которого следует из теоремы 2 , продолжим нулем вне $D_{1-\varepsilon}^{T}$ и обозначим через $u_{\varepsilon}$. Для него выполнено равенство

$$
\int_{Q^{T}}\left(\left(-u_{\varepsilon}\right) \varphi_{t}+\left\langle a_{\varepsilon}\left(u_{\varepsilon}\right)-u_{\varepsilon} \mathcal{G}\left(u_{\varepsilon}\right), d \varphi\right\rangle\right) d \nu d t=\int_{\mathbb{H}^{n}} u_{0} \varphi(x, 0) d \nu
$$

при всех $\varphi \in C_{0}^{1}\left(D_{r}^{T}\right)$. Подставляя сюда $\varphi=\alpha(t) w(x), \alpha \in C_{0}^{1}(0, T), w \in C_{0}^{1}\left(B_{1-\varepsilon}\right)$, устанавливаем, что функция $\int_{\mathbb{H}} u_{\varepsilon}(t) w d \nu$ абсолютно непрерывна.

Далее всюду будем предполагать, что $r<1-\varepsilon$.

Лемма 6. Пусть $\mathcal{F}$ - семейство неотрицательных функиий из $L_{\infty}\left(B_{r}\right), r \in(0,1)$, таких, чmo

$$
\|A(f)\|_{W_{p}^{1}\left(B_{r}\right)} \leq M \quad u \quad\|f\|_{L_{\infty}\left(B_{r}\right)} \leq M \quad \text { для любого } f \in \mathcal{F} .
$$

Тогда существует неубывающал функиия $\omega_{M}(\delta) \rightarrow 0$ при $\delta \rightarrow 0$ такал, что если для функций $f_{1}, f_{2} \in \mathcal{F}$ выполнено неравенство

$$
\int_{B_{r}}\left(A\left(f_{2}\right)-A\left(f_{1}\right)\right)\left(f_{2}-f_{1}\right) d x \leq \delta,
$$

mo $\left\|A\left(f_{2}\right)-A\left(f_{1}\right)\right\|_{L_{1}\left(B_{r}\right)} \leq \omega_{M}(\delta)$.

Доказательство леммы имеется в [3].

Лемма 7. Пусть $u_{\varepsilon} \in L_{\infty}\left(Q^{T}\right)$ - слабое решение задачи (11)-(13). Тогда существует функиия $C(r)$ такал, что при $r<1-\varepsilon$ справедлива оченка

$$
\left\|\nabla A_{\varepsilon}\left(u_{\varepsilon}\right)\right\|_{p, D_{r}^{T}} \leq C(r) .
$$

Доказательство. Из (25) и леммы 3 при $b(r)=r$ получаем соотношение

$$
\int_{Q^{T}}\left(\varphi_{t} \int_{u_{0}}^{u_{\varepsilon}} \xi(r) d r-\left\langle\left(\left|\nabla_{g} A_{\varepsilon}\left(u_{\varepsilon}\right)\right|_{g}^{p-2} \nabla_{g} A_{\varepsilon}\left(u_{\varepsilon}\right)-u_{\varepsilon} \mathcal{G}\left(u_{\varepsilon}\right)\right), d\left(\varphi \xi\left(u_{\varepsilon}\right)\right)\right\rangle\right) d \nu d t=0 .
$$

По теореме 2 имеем $0 \leq u_{\varepsilon} \leq \rho, \rho=M_{0} \exp (c T)$. Тогда $A_{\varepsilon}\left(u_{\varepsilon}\right) \leq A(\rho)+\rho, \varepsilon \in(0,1)$, и функцию $A_{\varepsilon}(s)$ временно можно считать ограниченной числом $A(\rho)+\rho$. Подставим в $(26)$ $\xi(s)=A_{\varepsilon}(s), \varphi \in C_{0}^{1}\left(D_{r^{\prime}}^{T}\right), r^{\prime}=1-(1-r) / 2$. Вводя обозначение $S_{\varepsilon}(v)=\int_{0}^{v} A_{\varepsilon}(s) d s$, получим

$$
\begin{gathered}
\int_{Q^{T}}\left(\varphi_{t}\left(S_{\varepsilon}\left(u_{\varepsilon}(t)\right)-S_{\varepsilon}\left(u_{0}\right)\right)-\varphi\left|\nabla_{g} A_{\varepsilon}\left(u_{\varepsilon}\right)\right|_{g}^{p}-\right. \\
\left.-\left\langle a_{\varepsilon}\left(u_{\varepsilon}\right), A_{\varepsilon}\left(u_{\varepsilon}\right) d \varphi\right\rangle+\left\langle u_{\varepsilon} \mathcal{G}\left(u_{\varepsilon}\right), d\left(\varphi A_{\varepsilon}\left(u_{\varepsilon}\right)\right)\right\rangle\right) d \nu d t=0 .
\end{gathered}
$$

В силу (6), (10) имеем

$$
\left|\left\langle u_{\varepsilon} \mathcal{G}\left(u_{\varepsilon}\right), d\left(\varphi A_{\varepsilon}\left(u_{\varepsilon}\right)\right)\right\rangle\right| \leq C\left(r^{\prime}, \rho\right)\left(\left|\varphi \nabla_{g} A_{\varepsilon}\left(u_{\varepsilon}\right)\right|_{g}+\left|\nabla_{g} \varphi\right|_{g}\right) .
$$


Положим $\varphi=z^{p}, z \in C_{0}^{1}\left(D_{r^{\prime}}^{T}\right), 0 \leq z \leq 1, z_{t} \leq 0$. Используя неравенство Юнга, запишем соотношения

$$
\left|\left\langle a_{\varepsilon}\left(u_{\varepsilon}\right), A_{\varepsilon}\left(u_{\varepsilon}\right) d \varphi\right\rangle\right| \leq C(\rho)\left|\left\langle a_{\varepsilon}\left(u_{\varepsilon}\right), z^{p-1} d z\right\rangle\right| \leq \frac{1}{2} \varphi\left|\nabla_{g} A_{\varepsilon}\left(u_{\varepsilon}\right)\right|_{g}^{p}+C\left|\nabla_{g} z\right|_{g}^{p} .
$$

Теперь из (27) нетрудно вывести неравенство

$$
\int_{Q_{r^{\prime}}^{T}} \frac{1}{4} \varphi\left|\nabla_{g} A_{\varepsilon}\left(u_{\varepsilon}\right)\right|_{g}^{p} d \nu d t \leq C(r, \rho)\left(1+\int_{Q_{r^{\prime}}^{T}}\left|\nabla_{g} z\right|_{g}^{p} d \nu d t\right) .
$$

Выбирая функцию $z$ так, чтобы $z=1$ при $x \in B_{r}$, выводим $\left\|\nabla_{g} A_{\varepsilon}\left(u_{\varepsilon}\right)\right\|_{p, Q_{r}^{T}} \leq C(r)$. Пользуясь (6), получим неравенство леммы.

Ниже используется обозначение $\left.\varphi\right|_{t} ^{\tau}=\varphi(\tau)-\varphi(t)$.

Лемма 8. Пусть $\eta(x) \in C_{0}^{1}\left(B_{r}\right)$ - неотрицательная функиия. Справедливо неравенство

$$
\left.\left.\int_{0}^{T-h} \int_{\mathfrak{B}_{\varrho}} \eta(x) u_{\varepsilon}\right|_{t} ^{t+h} A\left(u_{\varepsilon}\right)\right|_{t} ^{t+h} d \nu d t \leq C(r) h
$$

для всех $\varepsilon>0$ u $h \in[0, T / 2]$.

Доказательство. Подставим в $(25) \varphi=(\alpha(t) w(x))_{-h}, \alpha \in C_{0}^{1}(0, T), w(x) \in C_{0}^{1}\left(B_{r}\right)$. Пользуясь свойствами осреднения Стеклова имеем

$$
\int_{Q^{T}}\left(\left(u_{\varepsilon}\right)_{h t} \alpha w+\alpha\left\langle\left(a_{\varepsilon}\left(u_{\varepsilon}\right)\right)_{h}-\left(u_{\varepsilon} \mathcal{G}\left(u_{\varepsilon}\right)\right)_{h}, d w\right\rangle\right) d \nu d t=0 .
$$

После предельного перехода по последовательности $\alpha_{k} \rightarrow \delta\left(t-t_{0}\right)$ получим

$$
\left.h^{-1} \int_{\mathbb{H}^{n}} u_{\varepsilon}\right|_{t_{0}} ^{t_{0}+h} w d \nu=\int_{\mathbb{H}^{n}}\left\langle-\left(a_{\varepsilon}\left(u_{\varepsilon}\right)\right)_{h}\left(t_{0}\right)+\left(u_{\varepsilon} \mathcal{G}\left(u_{\varepsilon}\right)\right)_{h}\left(t_{0}\right), d w\right\rangle d \nu .
$$

Подставим в эту формулу $w(x)=\left.\eta(x) A\left(u_{\varepsilon}\right)\right|_{t_{0}} ^{t_{0}+h}, \eta(x) \in C_{0}^{1}\left(B_{r}\right)$. После интегрирования по $t_{0} \in(0, T-h)$ имеем

$$
\begin{gathered}
\left.\left.\int_{Q_{0}^{T-h}} \eta(x) u_{\varepsilon}\right|_{t} ^{t+h} A\left(u_{\varepsilon}\right)\right|_{t} ^{t+h} d \nu d t=h \int_{\mathbb{H}^{n}}\left\langle-\left(\left|\nabla_{g} A_{\varepsilon}\left(u_{\varepsilon}\right)\right|_{g}^{p-2} \nabla_{g} A_{\varepsilon}\left(u_{\varepsilon}\right)_{h}+\right.\right. \\
\left.\left.+\left(u_{\varepsilon} \mathcal{G}\left(u_{\varepsilon}\right)\right)_{h}\right), d\left(\left.\eta(x) A\left(u_{\varepsilon}\right)\right|_{t} ^{t+h}\right)\right\rangle d \nu d t \leq \\
\leq C h\left(\left\|\nabla_{g} A\left(u_{\varepsilon}\right)\right\|_{p, Q^{T}}+\left\|\nabla_{g} \eta\right\|_{p, Q^{T}}\right)\left(\left\|\nabla_{g} A_{\varepsilon}\left(u_{\varepsilon}\right)\right\|_{p, Q^{T}}+C_{G}(r)\right) .
\end{gathered}
$$

Отсюда следует неравенство (28).

Лемма 9. Семейство $\left\{z_{\varepsilon}=A\left(u_{\varepsilon}\right)\right\}$ компактно в $L_{1}\left(D_{r}^{T}\right)$.

Доказательство леммы имеется в [3] и опирается на леммы 7,8 .

В силу леммы 9 и возрастания функции $A$ можно выделить последовательность $\varepsilon_{k} \rightarrow 0$ при $k \rightarrow \infty$ такую, что $u_{\varepsilon_{k}} \rightarrow u$ п.в. в $B_{r}$. В силу произвольности $r \in(0,1)$ можно считать также, что $u_{\varepsilon_{k}} \rightarrow u$ п.в. в $B_{1}$. Это вместе с ограниченностью последовательности $u_{\varepsilon_{k}}$ в $L_{\infty}\left(Q_{r}^{T}\right)$ влечет сходимость $u_{\varepsilon_{k}} \rightarrow u L_{p}\left(Q_{r}^{T}\right)$ при любых $p \geq 1$ и $r<1$. Из леммы 7 следует ограниченность $\left\|a_{\varepsilon}\left(u_{\varepsilon}\right)\right\|_{\bar{p}, Q_{r}^{T}} \leq C$, поэтому можно считать, что $a_{\varepsilon_{k}}\left(u_{\varepsilon_{k}}\right) \rightarrow v$ слабо в $L_{\bar{p}}\left(Q_{r}^{T}\right)$ 
при $k \rightarrow \infty, \nabla_{g} A_{\varepsilon_{k}}\left(u_{\varepsilon_{k}}\right) \rightarrow \nabla_{g} A(u)$ слабо в $L_{p}\left(Q_{r}^{T}\right)$. Из леммы 1 также имеем равномерную сходимость

$$
\mathcal{G}\left(u_{\varepsilon_{k}}\right) \rightrightarrows \mathcal{G}(u) \text { в } B_{r}, k \rightarrow \infty .
$$

Поэтому $u_{\varepsilon_{k}} \mathcal{G}\left(u_{\varepsilon_{k}}\right) \rightarrow u \mathcal{G}(u)$ в $L_{p}\left(Q_{r}^{T}\right)$.

Предельный переход в $(25)$ при $\varepsilon_{k} \rightarrow 0$ приводит к соотношению

$$
\int_{Q^{T}}\left((-u) \varphi_{t}+\langle v-u \mathcal{G}(u), d \varphi\rangle\right) d \nu d t=\int_{\mathbb{H}^{n}} u_{0} \varphi(x, 0) d \nu
$$

при всех $\varphi \in C_{0}^{1}\left(Q^{T}\right)$. Применяя лемму 3 , из (29) получаем

$$
\int_{Q^{T}}\left(\varphi_{t} \int_{u_{0}}^{u} \xi(r) d r-\langle v-u \mathcal{G}(u), d(\varphi \xi(u))\rangle\right) d \nu d t=0 .
$$

Выбирая в $(30) \varphi=\alpha(t) \eta(x), \alpha \in C_{0}^{1}(0, T), \xi(s)=A(s)$, имеем

$$
\int_{Q^{T}}\left(\eta(x) \alpha_{t} S_{0}(u(t))-\langle v-u \mathcal{G}(u), \alpha d(\eta(x) A(u))\rangle\right) d \nu d t=0,
$$

где $S_{0}=\left.S_{\varepsilon}\right|_{\varepsilon=0}$. Полагая в $(27) \varphi=\alpha \eta(x)$, и переходя к пределу по $\varepsilon_{k} \rightarrow 0$, получим

$$
\begin{aligned}
\int_{Q^{T}}\left(\eta(x) \alpha_{t} S_{0}(u(t))\right. & +\langle u \mathcal{G}(u), d(\alpha \eta(x) A(u))\rangle-\langle v, \alpha A(u) d \eta(x)\rangle) d \nu d t= \\
& =\lim _{k \rightarrow \infty} \int_{Q^{T}} \alpha \eta(x)\left|\nabla_{g} A_{\varepsilon}\left(u_{\varepsilon}\right)\right|_{g}^{p} d \nu d t .
\end{aligned}
$$

Сравнивая последнее с (31), устанавливаем, что

$$
\lim _{k \rightarrow \infty} \int_{Q^{T}} \alpha \eta(x)\left|\nabla_{g} A_{\varepsilon}\left(u_{\varepsilon}\right)\right|_{g}^{p} d \nu d t=\int_{Q^{T}} \alpha \eta(x)\langle v, d A(u)\rangle d \nu d t .
$$

В силу монотонности $a_{\varepsilon}\left(u_{\varepsilon}\right)$ для $w \in W_{p}^{1,0}\left(Q^{T}\right)$ имеем

$$
\begin{gathered}
0 \leq \int_{Q^{T}} \alpha \eta(x)\left\langle\left(a_{\varepsilon}\left(u_{\varepsilon}\right)-\left|\nabla_{g} w\right|_{g}^{p-2} \nabla_{g} w\right), d\left(A_{\varepsilon}\left(u_{\varepsilon}\right)-w\right)\right\rangle d \nu d t=\int_{Q^{T}} \alpha \eta(x)\left\langle a_{\varepsilon}\left(u_{\varepsilon}\right), d A_{\varepsilon}\left(u_{\varepsilon}\right)\right\rangle d \nu d t- \\
-\int_{Q^{T}} \alpha \eta(x)\left\langle\left(a_{\varepsilon}\left(u_{\varepsilon}\right), d w\right)\right\rangle d \nu d t-\int_{Q^{T}} \alpha \eta(x)\left\langle\left(\left|\nabla_{g} w\right|_{g}^{p-2} \nabla_{g} w, d\left(A_{\varepsilon}\left(u_{\varepsilon}\right)-w\right)\right)\right\rangle d \nu d t .
\end{gathered}
$$

Переходя к пределу по $\varepsilon_{k} \rightarrow 0$ и используя (32), получим

$$
0 \leq \int_{Q^{T}} \alpha \eta(x)\left\langle\left(v-\left|\nabla_{g} w\right|_{g}^{p-2} \nabla_{g} w, d(A(u)-w)\right)\right\rangle d \nu d t .
$$

Подставляя сюда $w=A(u)-\varepsilon z, z \in W_{p}^{1,0}\left(Q^{T}\right)$, деля на $\varepsilon$, и переходя к пределу при $\varepsilon \rightarrow 0$, находим, что

$$
0 \leq \int_{Q^{T}} \alpha \eta(x)\left\langle\left(v-\left|\nabla_{g}(A(u))\right|_{g}^{p-2} \nabla_{g}(A(u)), d z\right)\right\rangle d \nu d t
$$


при любом $z$ и любом $\eta \in C_{0}^{1}\left(B_{r}\right), \alpha \in C_{0}^{1}(0, T), \alpha \geq 0$. Это возможно лишь при

$$
\left\langle\left(v-\left|\nabla_{g}(A(u))\right|_{g}^{p-2} \nabla_{g}(A(u)), d z\right)\right\rangle \equiv 0 .
$$

Тогда (29) означает, что $u$ - слабое решение задачи (4), (5).

Автор выражает искреннюю благодарность $\Phi$. Х. Мукминову за обсуждение результатов работы и полезные замечания.

\section{ЛитератУРА}

[1] Punzo F. Well-posedness of the Cauchy problem for nonlinear parabolic equations with variable density in the hyperbolic space, Nonlinear Diff. Equat. and Appl.19, 485-501 (2012).

[2] Bertozzi A., Slepcev D. Existence and Uniqueness of Solutions to an Aggregation Equation with Degenerate Diffusion, Comm. Pur. Appl. Anal., 9 (6), 1617-1637 (2010).

[3] Вильданова В.Ф. Существование и единственность слабого решения нелокалъного уравнения агрегации с вырождающейсл дифбузией общего вида, Матем. сб., 209 (2), 66-81 (2018).

[4] Kamin Sh., Pozio M.A., Tesei A. Admissible conditions for parabolic equations degenerating at infinity, Алгебра и анализ, 19 (2), 105-121 (2007).

[5] Carrillo J., Wittbold P. Uniqueness of renormalized solutions of degenerate elliptic-parabolic problems, J. Diff. Equat., 156, 93-121 (1999).

[6] Соболев С.Л. Некоторие применения функционалъного анализа в математической физике (Наука, М., 1988).

[7] Вильданова В.Ф., Мукминов Ф.Х. Существование слабого решения интегро-дифберенииального уравнения агрегации, Совр. пробл. матем. Фундамент. направления, 63 (4), 557-572 (2017).

[8] Вильданова В.Ф. Существование и единственность слабого решения интегро-дифференциалъного уравнения агрегации на римановом многообразии, Матем. сб., 211 (2), 74-105 (2020).

[9] Мукминов Ф.Х. Единственность ренормализованного решения эллиптико-параболической задачи в анизотропных пространствах Соболева-Орлича, Матем. сб., 208 (8), 1187-1206 (2017).

\section{Венера Фидарисовна Вильданова}

Башкирский государственный педагогический университет им. М. Акмуллы, ул. Октябрьской революции, д.3а, г. Уфа, 450008, Россия,

e-mail : gilvenera@mail.ru

\section{V.F. Vil'danova}

\section{Existence of a solution to the Cauchy problem for the aggregation equation in hyperbolic space}

Abstract. In hyperbolic space, we consider the Cauchy problem for the aggregation equation. Nonnegative initial function limited and summable. The existence of a weak solution is proved on small time interval. In the case where the kernel of the integral operator is smooth and rapidly decreases at infinity, the existence of a bounded solution on an arbitrary interval of time is proved.

Keywords: the aggregation equation, solution existence, hyperbolic space.

\section{Venera Fidarisovna Vil'danova}

Bashkir State Pedagogical University of M. Akmulla, 3a Oktyabrskoj Revoljucii str., Ufa, 450008 Russia,

e-mail: gilvenera@mail.ru 